\title{
Le canal de Suez face aux évolutions de la marine marchande : un siècle et demi de défis techniques
}

The Suez Canal in front of the evolution of the merchant navy: one century and a half of technical challenges

\section{Caroline Piquet}

\section{OpenEdition}

Journals

Édition électronique

URL : http://journals.openedition.org/artefact/4151

DOI : $10.4000 /$ artefact. 4151

ISSN : 2606-9245

Éditeur :

Association Artefact. Techniques histoire et sciences humaines, Presses universitaires du Midi

Édition imprimée

Date de publication : 15 octobre 2019

Pagination : 175-191

ISBN : 978-2-8107-0650-1

ISSN : 2273-0753

\section{Référence électronique}

Caroline Piquet, «Le canal de Suez face aux évolutions de la marine marchande : un siècle et demi de défis techniques », Artefact [En ligne], 10 | 2019, mis en ligne le 06 août 2020, consulté le 27 novembre 2020. URL : http://journals.openedition.org/artefact/4151; DOI : https://doi.org/10.4000/ artefact. 4151

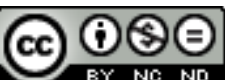

Artefact, Techniques, histoire et sciences humaines est mise à disposition selon les termes de la Licence Creative Commons Attribution - Pas d'Utilisation Commerciale - Pas de Modification 4.0 International. 


\section{Le canal de Suez face aux évolutions de la marine marchande : un siècle et demi de défis techniques}

\section{Caroline Piquet}

\section{Résumé}

Le canal de Suez depuis son ouverture à la navigation en 1869 représente une réalisation technique en évolution permanente, soumise aux évolutions de la marine marchande au cours des deux derniers siècles. Au xIx siècle, il constitue une étape décisive dans l'histoire des travaux publics en révolutionnant les techniques de dragage. L'essor du commerce maritime dans le dernier quart du $\mathrm{xIX}$ siècle pousse les ingénieurs à concevoir plusieurs programmes d'adaptation du canal en fonction de la modernisation des flottes. Au siècle suivant, l'apparition des tankers et porte-conteneurs toujours plus imposants impose de nouveaux travaux au canal afin que celui-ci puisse conserver son rang parmi les premières routes maritimes mondiales. Au début $d u x x l^{e}$ siècle, avec les travaux de 2015, cette préoccupation demeure essentielle pour l'Égypte qui entend grâce au canal consolider sa place dans le commerce entre l'Europe et l'Asie.

\section{Mots-clés}

canal, route maritime, marine marchande, commerce maritime, techniques de dragage, travaux publics, Égypte

95 Caroline Piquet, « Le canal de Suez face aux évolutions de la marine marchande : un siècle et demi de défis techniques », Artefact, 10, 2019, p. 175-191. 


\section{The Suez Canal in front of the evolution of the merchant navy: one century and a half of technical challenges}

\section{Abstract}

Since its opening to navigation in 1869, the Suez Canal has represented a constantly evolving technical realization, subject to the evolutions of merchant navy during the last two centuries. In the 19th century, the canal constituted a decisive step in the history of public works by revolutionizing dredging techniques. The growth of maritime trade in the last quarter of the 19th century prompted the engineers to design several programs of adaptation of the canal according to the modernization of the fleets. In the following century, the emergence of tankers and container ships forced new works on the canal so that it could maintain its rank among the world's leading shipping routes. At the beginning of the 21st century, with the works of 2015, this concern remains essential for Egypt that intends through the canal, to consolidate its place in trade between Europe and Asia.

\section{Keywords}

canal, sea route, shipping, maritime trade, dredging techniques, publics works, Egypt 


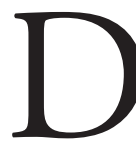

u point de vue de l'histoire des techniques, le canal de Suez est surtout célèbre pour la période du chantier (1859-1869), présentée comme un moment fort dans l'évolution des travaux publics. Toutefois, cet épisode ne représente que le début d'une adaptation continue à la marine marchande jusqu'aux travaux de 2015. Au XIx siècle, le canal s'inscrit dans un contexte technique inédit avec la transition de la navigation à voile à la navigation à vapeur ; la marine marchande se mécanise progressivement, ce qui permet au navire de gagner en puissance, en capacité de charge et en vitesse. L'utilisation de la vapeur permet aussi de concevoir des lignes plus fiables et plus régulières, favorisant l'essor des grandes compagnies de navigation et encourageant les armateurs à la modernisation de leurs équipements. Au xx siècle, le canal doit faire face à de nouvelles transformations de la marine marchande, avec l'apparition des navires spécialisés et surtout avec l'augmentation des tonnages qui oblige à considérer régulièrement les dimensions du canal. Ce processus se déroule dans un contexte toujours plus compétitif où le canal de Suez doit convaincre les armateurs, au prix d'investissements lourds mais nécessaires pour rester parmi les premières voies maritimes mondiales.

Pour reconstituer l'histoire technique du canal, le fonds d'archives de la Compagnie du canal de Suez qui a géré la voie maritime jusqu'en 1956 est essentiel, constitué de maquettes, de rapports et de publications régulières sur les programmes d'entretien ${ }^{1}$. Après 1956 , les rapports annuels de l'organisme d'État chargé de la gestion du canal, la Suez Canal Authority, fournissent les principales sources d'information, avec les publications de la Banque internationale pour la reconstruction et le développement ${ }^{2}$.

\footnotetext{
1. Archives privées de la Compagnie universelle du canal maritime de Suez (CUCMS), Centre des archives du monde du travail, Roubaix ; Le Canal de Suez, bulletin décadaire de la compagnie universelle du canal de Suez, 1872-1939 ; Le Canal de Suez. Documents statistiques, 1927 ; Le Canal de Suez. Documents statistiques, 1936-1937 ; Le Canal de Suez. Notes, tableaux et planches, 1908, 1937, 1950. Notons aussi la publication par la Compagnie du mensuel Le Canal, de 1952 à 1956, journal d'entreprise, riche en articles techniques sur la voie maritime.

2. Suez Canal Authority, 1991-2016 (les plus récents sont consultables sur le site www.suezcanal. gov.eg) ; Loan Agreement, 1959 ; Loan Agreement, 1974. Ces sources sont analysées dans Piquet, 2009, réédité dans Piquet, 2018.
} 


\section{Le canal et les innovations du transport maritime au XIX ${ }^{\mathrm{e}}$ siècle}

\section{Le chantier du creusement : une grande aventure technique}

Le chantier du canal de Suez représente une étape importante dans l'histoire du génie civil ${ }^{3}$. L'entreprise chargée du creusement, la Compagnie universelle du canal maritime de Suez, commence en 1859 les travaux avec une main-d'œuvre locale dans le cadre de la corvée. En 1864, avec la fin de la corvée imposée par l'Empire ottoman, l'entreprise décide de s'équiper en machines innovantes reposant sur la force de la vapeur et investit massivement dans le rail pour faciliter le transport des déblais et des matériaux. La drague devient alors la machine emblématique du chantier de Suez. Ces nouveaux engins sont actionnés par la vapeur et sont dotés d'une structure en fer, ce qui les rend bien plus puissants que ceux utilisés jusque-là sur d'autres chantiers. L'évacuation des déblais est aussi sensiblement améliorée avec la mise au point de dragues à long couloir et d'élévateurs actionnés par la vapeur ainsi qu'avec la généralisation du rail sur le chantier. De grandes améliorations sont aussi apportées à la technique de terrassement des zones rocheuses grâce aux excavateurs Couvreux, du nom de son entrepreneur, dont une quinzaine est construite pour le chantier du canal.

Par ailleurs, le chantier du canal est un terrain d'expérimentation de nouveaux procédés et de perfectionnement des matériaux, comme le ciment aggloméré breveté en 1855 par François Coignet et utilisé pour la construction des jetées et l'édification du phare de Port-Saïd. C'est à Port-Saïd également que l'entreprise Dussaud réalise des blocs artificiels en ciment à partir de base de sable et de chaux. On parle alors de " chantier dans le chantier » avec la fabrication de 30000 blocs de plus de 20 tonnes destinés à la construction de la digue.

Lors de l'inauguration en novembre 1869, les travaux ne sont pas encore terminés ; au niveau des zones rocheuses le tirant d'eau nécessaire aux navires de l'époque (22 pieds) n'est réalisé que quelques semaines avant l'ouverture du canal à la navigation. Le jour de l'inauguration, Le Péluse,

3. Montel, 1998. 
navire des Messageries impériales de 2000 tonnes $^{4}$, passe tout juste; pour d'autres bâtiments, la charge du bateau est déplacée vers l'avant afin de diminuer le tirant d'eau à l'arrière. Les travaux se poursuivent au début des années 1870 afin d'élargir des courbes et d'approfondir la cuvette sur une soixantaine de kilomètres. De plus, dès 1876, avec le rapide essor de la navigation, de nouvelles améliorations s'imposent dans le tracé des courbes et de l'élargissement du chenal au niveau de plusieurs sections. Ces améliorations doivent inévitablement intégrer l'évolution des marines marchandes mondiales. Pour ce faire, une concertation des milieux concernés au sein d'un organisme chargé des grands programmes de travaux s'avère indispensable.

\section{Le rôle de la Commission internationale des travaux}

Ferdinand de Lesseps, président-directeur de la Compagnie du canal de Suez, crée en 1884 la Commission internationale des travaux, un organisme qu'il veut international dans le même esprit que les commissions qu'il avait réunies lors du chantier, avec la collaboration d'ingénieurs et d'armateurs de toutes nations. En dix-huit ans, il suscite six commissions : la première Commission internationale du canal de Suez, chargée de rendre le rapport sur le projet du trajet, voit le jour en 1855 ; durant les premiers mois du chantier, un conseil supérieur de travaux est constitué, il devient, en 1861, la Commission consultative des travaux ; en 1865, deux nouvelles commissions se réunissent afin de se prononcer sur l'ensemble des travaux ; en 1873, sous la pression des armateurs, la Commission du tonnage réunit quinze spécialistes pour trancher la question des tarifs ; enfin, en 1884, la Commission consultative internationale doit se prononcer sur les améliorations régulières du canal. À partir de 1887 et jusqu'en 1956, sauf périodes de guerre, l'organisme tient une session annuelle de façon à assurer l'adaptation du canal à l'évolution de la marine. Cet organisme est composé de douze à vingt-deux experts hautement qualifiés, ingénieurs en travaux maritimes associés à quelques marins appartenant suivant la période à six, sept ou huit pays différents.

4. Il s'agit de la tonne TPL « tonne de port en lourd ». Cette unité de mesure est utilisée pour calculer le tonnage maximum qu'un bateau peut charger (égal au poids total du navire chargé au maximum moins son poids à vide). 
Quinze après l'inauguration, la Commission des travaux se prononce pour un programme d'élargissement destiné à adapter la profondeur du chenal aux nouveaux steamers qui présentent désormais des tirants d'eau de 7,5 à 8 mètres. Les travaux d'approfondissement s'achèvent en 1890 et permettent d'atteindre une profondeur de 8,5 mètres sur tout le parcours contre les 8 mètres initiaux. Huit années sont encore nécessaires pour porter la largeur de la cuvette de 22 à 37 mètres. Le croisement des convois a été amélioré grâce au creusement tous les 10 kilomètres de zones aménagées pour garer les navires. Mais un programme est-il à peine achevé que l'évolution des bateaux nécessite de nouveaux élargissements : en 1900, les gares sont agrandies, la cuvette est approfondie et le tirant d'eau maximum autorisé des navires passe à 8 mètres, puis à 8,5 mètres en 1908. Quant à la largeur de la cuvette mesurée à la surface de l'eau, elle atteint désormais 80 à 120 mètres selon le tracé, contre 54 à 100 mètres en $1869^{5}$.

Outre les grands programmes d'amélioration décidés par la Commission des travaux, la Compagnie de Suez se charge des travaux d'entretien réguliers dont les opérations principales sont le dragage, l'entretien des berges et le balisage. Le dragage représente l'activité essentielle des services de la Compagnie du fait de l'ensablement naturel de la cuvette, en moyenne de 40 centimètres par an. De l'ouverture à la nationalisation de 1956, les dragages effectués dans le cadre des travaux d'entretien et d'élargissement sont estimés à plus de 400 millions de mètres cubes ${ }^{6}$. Depuis l'époque du percement, l'entreprise a développé une grande expérience en ce domaine et poursuit son équipement : aux dragues à godets utilisées lors du chantier, elle ajoute des machines plus performantes avec la drague aspiratrice qui, comme son nom le suggère, aspire les terrains meubles; la Compagnie acquiert aussi la drague à godets refouleuse, construite au Havre par la société des Forges et chantiers de la Méditerranée, qui creuse jusqu’à 13,5 mètres sous l'eau. La protection des berges constitue l'autre grande préoccupation des services d'entretien. Soumises à l'érosion provoquée par l'eau de mer et les remous engendrés par le passage des navires, les berges s'effondrent régulièrement. Au moment de l'inauguration, les défenses par enrochement demeurent faibles et, avec la mise en exploitation du canal, l'érosion des berges apparait bien vite comme une difficulté majeure. Les perrés (ou murs de soutènement) sont étendus, mais il convient de les

5. Le Canal de Suez. 1908, 1937, 1950 ; Piquet, 2018, p. 110.

6. Le Canal, $\mathrm{n}^{\circ} 12$, juin 1954, p. 2. 
consolider régulièrement, voire de les reconstruire après les travaux d'élargissement. D’autres méthodes sont aussi testées : près de Suez, les revêtements maçonnés font leur apparition à la fin du XIX ${ }^{\mathrm{e}}$ siècle; dans la section centrale d'Ismaïlia, des tamaris, des filaos et des roseaux sont plantés le long des berges, mais sans franc succès. Enfin, une centaine d'ouvriers sont affectés au balisage et à l'entretien des bouées. Le canal est balisé sur tout son parcours de kilomètre en kilomètre dans les parties étroites et de 300 en 300 mètres dans la partie concave des courbes au moyen de bouées biconiques. Les berges sont aussi bordées de poteaux indiquant la présence de bancs rocheux et délimitant les zones de garage ou d'amarrage ${ }^{7}$.

Cependant, malgré ses aménagements constants, le chenal apparaît dès la fin de la Première Guerre mondiale trop étroit et trop peu profond pour accueillir les nouveaux navires qui sont désormais deux fois plus larges et quatre plus longs que les premiers steamers du Xix ${ }^{e}$ siècle pour lesquels le canal avait été conçu.

\section{S'affirmer comme une des premières voies mondiales du transport maritime (1914-1956)}

\section{S'adapter aux mutations techniques de l'entre- deux-guerres}

Les années 1920 et 1930 se caractérisent par la motorisation des flottes et l'apparition des navires spécialisés. Le diesel s'impose progressivement ; en 1931, la part de la navigation à moteur dans le tonnage total de Suez passe à $25 \%$ alors que sa proportion dans l'effectif mondial s'établit à $13 \%$. En outre, la rude concurrence entre les armateurs accélère la modernisation des navires ; les navires à fort tonnage s'imposent afin d'obtenir de meilleurs rendements et de réduire les coûts. Les années 1920 correspondent au développement des paquebots spécialisés, avec les charbonniers qui se transforment en minéraliers, l'apparition de navires équipés d'installations frigorifiques pour le transport des denrées alimentaires et le déploiement des bateaux-citernes réservés au transport de l'huile végétale.

7. Le Canal, $\mathrm{n}^{\circ} 11$, avril 1954, p. 7-12 et n²4, juin 1956, p. 6-13. 
Parmi ces derniers, se distinguent les navires-citernes destinés au transport du pétrole, appelés aussi tankers, dont l'essor est lié à la généralisation du moteur diesel et à la découverte de gisements pétrolifères. Le golfe Persique est devenu une zone de production importante depuis la découverte en 1908 des gisements d'Abadan exploités par l'Anglo-Persian Oil Company. Pour acheminer sa marchandise vers la raffinerie de Skewen au pays de Galles, cette dernière crée en 1915 la British Tanker qui effectue déjà une quarantaine de traversées annuelles dans le canal au milieu des années $1920^{8}$.

Après la Seconde Guerre mondiale, la marine de guerre, avec les cuirassés et les porte-avions ainsi que la marine marchande connaissent une étonnante croissance de leurs capacités de tonnage. La flotte pétrolière connaît également un essor considérable : au début des années 1950, elle constitue $60 \%$ des usagers du canal, ce qui correspond à près de quatre millions de tonnes transitant dans le sens sud-nord. Le pétrole a des effets sur l'évolution du trafic - le trafic par jour double entre 1938 et 1954 - mais il en a aussi sur l'évolution des flottes avec le passage à la motorisation des navires : la proportion du tonnage mondial utilisant des combustibles liquides en 1953 est de $87 \%$; le charbon est tombé à $13 \%$ (contre $97 \%$ en 1914) ${ }^{9}$. Les navires sont plus nombreux et plus imposants avec la généralisation après la guerre des navires de 10000 à 15000 tonnes. Au début des années 1950, apparaissent aussi les supertankers de plus de 28000 tonnes, comme quatre des navires de l'Anglo-Saxon Petroleum Company qui présentent des tirants d'eau de 10,36 mètres ou les bâtiments français Bérénice et Bethsabee, construits pour un transport à pleine charge de 31400 tonnes, trop lourds pour franchir le canal mais qui ont anticipé son approfondissement ${ }^{10}$.

\section{Les programmes d'élargissement}

Sous la pression constate de la flotte marchande, la Compagnie de Suez enchaîne les programmes de travaux de la voie maritime. Huit programmes

8. Piquet, 2018, p. 211.

9. Rapport du supplément du Lloyd's Register of Shipping, édition 1954, cité dans Le Canal, n 11, avril 1954, p. 26-27.

10. Piquet, 2018, p. 214. 
se succèdent de 1885 à $1956^{11}$ : le premier avait consisté à porter la profondeur de la cuvette 8,5 mètres ; les quatre programmes suivants, en 1901, 1903, 1908 et 1912, poursuivent l'élargissement et le creusement de la cuvette qui atteint 12 mètres de profondeur à la veille de la Première Guerre mondiale, pour une largeur de 47 mètres. Le canal souffre des conséquences du conflit ; les épaves et les mines doivent être nettoyées et le temps de traversée est passé de seize heures en 1913 à plus de vingttrois heures en 1918. Trois ans plus tard, la Compagnie lance un sixième programme de travaux. Ralenti par la crise économique mondiale, il arrive à son terme en 1934 ; avec une cuvette portée à 13 mètres de profondeur, le tirant d'eau autorisé est désormais de 33 pieds (10,06 mètres), ce qui permet d'accueillir la grande majorité des navires en circulation ; seuls onze navires parmi la flotte mondiale affichent un tirant d'eau supérieur à celui-ci. Enfin, le trafic est amélioré avec une baisse notable du temps de traversée réduit à moins de quatorze heures.

La Seconde Guerre mondiale correspond à un nouvel épisode de crise avec l'endommagement de la structure du canal du fait des mines et des bombardements. Les berges ont également beaucoup souffert du conflit : les murs de soutènement s'effondrent en de nombreux endroits et les fers de soutien se déchaussent. Avec la reprise du trafic et le passage de tankers plus rapides et plus imposants, la dégradation des berges s'accélère, phénomène que les ingénieurs tentent de freiner avec des protections en béton armé ou en planches métalliques. Néanmoins, le plus préoccupant demeure la hausse du trafic pétrolier qui conduit la Compagnie à adopter un septième programme en 1947 destiné à approfondir la cuvette à 14 mètres ; il est également envisagé de faciliter les croisements des convois venus du nord et du sud par la construction entre Port-Saïd et les lacs Amer d'une branche dérivée de 10 kilomètres, parallèle au canal. Ces travaux nécessitent une modernisation de l'équipement de l'entreprise qui fait l'acquisition de dragues plus performantes que les traditionnelles dragues à godets, comme la Paul Solente, construite à Nantes par les Ateliers et chantiers de Bretagne dont les moteurs diesel permettent de creuser jusqu'à 16 mètres de profondeur ou la Louis-Perrier, drague aspiratrice à désagrégateur livrée par les chantiers Werf Gusto de Schiedam en Hollande.

11. Piquet, 2018, p. 216-219. 
Alors que s'achève le septième programme en 1954, le canal est confronté à l'apparition des supertankers de plus de 30000 tonnes avec des capacités de chargement à 36 pieds. Un an plus tard, un nouveau programme est lancé pour creuser la cuvette et améliorer la fluidité des traversées dont le temps s'est rallongé du fait de l'augmentation du trafic, en retombant à la moyenne des années 1920 ( 15 heures) contre 13 heures 23 en 1938. De plus, pour la première fois, le grand lac Amer doit subir des dragages pour répondre aux dimensions des nouveaux navires et le doublement du canal s'accélère avec le creusement de deux nouvelles dérivations.

La crise de Suez qui suit l'annonce de la nationalisation de la Compagnie du canal le 26 juillet 1956 par le colonel Nasser met fin à la gestion européenne du canal. Si cela correspond à une rupture politique décisive, en revanche, du point de vue technique et commercial, la continuité demeure. Les dégâts des bombardements d'octobre 1956 sont vite dégagés : grâce à une aide financière et à une assistance technique de l'Organisation des Nations unies, le canal est à nouveau ouvert à la navigation au printemps 1957.

\section{L'évolution du canal sous gestion égyptienne (1956-2015)}

\section{Une gestion perturbée par les conflits régionaux}

Après la nationalisation de la Compagnie de Suez, l'entretien du canal représente un enjeu majeur pour l'Égypte. L'argument des Britanniques et des Français pour dénoncer le geste de Nasser reposait en effet sur l'idée que le pays serait incapable d'assurer son entretien, menaçant ainsi l'ensemble du transport maritime. En 1957, l'Égypte doit prouver le contraire : la Suez canal Authority, organisme d'État bénéficiant d'une autonomie administrative, est créée à cette fin. $25 \%$ de ses recettes sont reversées à un fonds d'équipement et de modernisation et, dès la réouverture du canal en avril 1957, les travaux entrepris par l'ancienne direction sont poursuivis. À l'automne 1958, les autorités lancent le plan Nasser, premier plan égyptien, le neuvième depuis l'ouverture : grâce au prêt de la Banque internationale pour la reconstruction et le développement (BIRD) de 56,5 millions de dollars, la cuvette est approfondie à 15,5 mètres, ce qui permet d'accueillir 
à la veille de la guerre des Six Jours des navires de 80000 tonnes avec un tirant d'eau de 38 pieds. Cependant la troisième guerre israélo-arabe vient perturber la troisième phase des travaux ${ }^{12}$.

La guerre de 1967 est un drame pour le canal puisque celui-ci est transformé en frontière militaire et demeure fermé à la navigation durant huit ans, sans bénéficier d'aucun entretien et soumis aux opérations militaires. Les conséquences sont désastreuses sur l'ensablement de la cuvette et l'effondrement des berges, sans compter les appareils coulés qui empêchent la navigation. De plus, le transport des hydrocarbures s'est réorganisé autour des oléoducs et des navires-citernes supergéants dont les dimensions ne permettent plus le passage par Suez. Seulement $23 \%$ de la flotte pétrolière peut encore emprunter le canal en 1975. L'Égypte doit par conséquent engager de gros travaux pour rattraper son retard sur les évolutions de la marine marchande ; elle obtient de la BIRD un prêt de 50 millions de dollars au taux de $8 \%{ }^{13}$. La SCA bénéficie aussi du concours de huit entreprises étrangères qui s'ajoutent à une vingtaine d'entreprises nationales. La planification des travaux est confiée à de grandes sociétés d'hydraulique françaises et britanniques, la Sogreah, Maunsell et Coopers \& Lybrand, alors qu'Américains et Japonais se chargent des branches de dérivation.

Le travail effectué est colossal : commencé en 1975, il s'achève cinq ans plus tard avec plus de 595 millions de mètres cubes dragués, plus que pendant toute la période précédente, chantier compris ${ }^{14}$. L'approfondissement de la cuvette permet le passage des navires de plus de 150000 tonnes à pleine charge, avec un tirant d'eau autorisé de 53 pieds (16 mètres). En outre, le canal a été doublé en quatre sections afin de faciliter les convois en double sens ${ }^{15}$.

Toutefois, le canal peine à retrouver la place qu'il occupait dans le commerce maritime avant la guerre des Six Jours. La compétitivité de la voie égyptienne est sérieusement entamée par le développement de ses concurrents. Les itinéraires du canal de Panama et du cap de Bonne Espérance sont devenus des rivaux plus dangereux qu'au milieu du siècle en raison

12. Piquet, 2018, p. 271.

13. Loan agreement, 1974.

14. De l'ouverture à la nationalisation, ils sont estimés à 400 millions de mètres cubes auxquels il faut ajouter les 74 millions de mètres cubes du chantier de creusement. Le Canal, n ${ }^{\circ}$ 2, juin 1954. 15. Piquet, 2018, p. 289. 
de l'incapacité technique du canal à accueillir les tankers géants. De plus, les limites techniques de la voie d'eau demeurent préoccupantes : environ la moitié du pétrole chargé sur tankers ne peut plus l'emprunter. Les porte-conteneurs, dynamisés par l'essor des échanges entre l'Asie et l'Europe, connaissent une évolution remarquable : en trente ans, leur taille moyenne passe de 2000 à 6000 evp. Leurs dimensions rendent la traversée difficile et le croisement quasi impossible : en 1986, la voie d'eau ne peut organiser que trois convois par 24 heures contre quatre avant 1956. La SCA lance au début des années 1990 le second programme de travaux depuis la nationalisation (le dixième depuis l'inauguration) afin de porter le tirant d'eau à 56 pieds ( 17 mètres), permettant le passage des navires de 160000 tonnes à pleine charge et de 250000 tonnes à vide. La SCA en profite pour moderniser ses installations avec l'informatisation des données, les radars et les systèmes de radio. D’autre part, de nouvelles générations de porte-conteneurs apparaissent avec des bâtiments de 12000 à 15000 evp, ce qui pousse la SCA à engager au début des années 2010 de nouveaux travaux d'approfondissement de la cuvette pour la porter à 24 mètres $^{16}$.

\section{5 : le « nouveau canal de Suez »}

Les travaux des années 2000, bien qu'ambitieux, ne parviennent toutefois pas à relever pleinement le défi lancé par l'essor des tonnages de la marine marchande. À la même époque, les travaux d'élargissement du canal de Panama, commencés en 2007, raniment la concurrence avec Suez. De plus, l'Égypte veut redevenir la principale route pétrolière entre l'Europe et le golfe Persique en récupérant les tankers géants passant par le cap de Bonne Espérance ainsi que le pétrole transitant par les oléoducs. C'est ainsi que le président de la République d'Égypte, le maréchal Sissi, annonce en 2014 les travaux du «nouveau canal de Suez ». Ce terme désigne en réalité le doublement d'une partie de la structure initiale de la voie maritime. Le projet représente un chantier de 72 kilomètres, composé de deux tronçons de 35 et 37 kilomètres. Sur la première partie, il s'agit de creuser une nouvelle voie de navigation à côté de la première ; sur l'autre partie, le chenal doit être élargi, approfondi et nettoyé. Le canal peut accueillir des

16. Piquet, 2018, p. 296. 
navires de 240000 tonnes mais ces navires larges en surface de 40 mètres, voire de 50 mètres pour les plus gros, ne permettent plus les croisements dans le canal autrement que dans les zones de dérivation. Celles-ci ressemblent à des bretelles d'autoroute où les bateaux peuvent circuler dans les deux sens. Le canal est déjà doublé en quatre endroits pour permettre aux navires de se croiser : à la dérivation Ballah de 9 kilomètres achevée en 1951, s'ajoutent les dérivations de Port-Saïd de 36,5 kilomètres et de Kabret, de 27,5 kilomètres, toutes deux terminées en 1980. Enfin, la dérivation de Timsah, commencée en 1975, s'étend sur 5 kilomètres au niveau du lac du même nom. À la veille des travaux de 2014, le canal est doublé sur $40 \%$ de sa longueur ; il s'agit de porter ce taux à plus de $58 \%$.

Le chantier est organisé en deux parties : d'un côté, le chantier de la création d'une nouvelle voie d'eau de 35 kilomètres parallèle au canal d'origine au nord du lac Timsah ; de l'autre, l'approfondissement et l'élargissement de la voie historique sur un tronçon de 37 kilomètres situé au niveau du lac Amer. La zone du premier chantier se situe à 24 mètres au-dessus du niveau de la mer : mission est donc confiée à l'armée égyptienne d'araser le relief pour préparer le terrain et permettre les futurs travaux de creusement. Bulldozers, bennes et camions dégagent 250 millions de mètres cubes de sable, de roche et de terre. Ensuite, la zone est inondée afin que les dragues puissent y flotter, pour ensuite retirer la même quantité de sable désormais située sous l'eau ${ }^{17}$.

Entrent alors en scène les opérations de dragages. En 2014, les autorités de Suez font appel à deux groupements d'entreprises de dragage ; le premier est chargé de creuser le nouveau tronçon, le second doit élargir le chenal historique. Une contrainte forte s'impose à ce dernier chantier : exécuter les travaux sans interrompre la circulation. À ce défi, s'ajoute la contrainte de temps qui de cinq ans a été ramenée à trois ans, puis à un an : pour les équipes techniques, le calendrier devient un challenge de taille. L'autre défi consiste en l'acheminement du matériel et du personnel. En l'espace de quelques mois, il faut faire venir dragues, remorqueurs, conduites,

17. Site officiel de la Suez Canal Authority : www.suezcanal.gov.eg 
pontons. Le personnel vient aussi du monde entier : vingt-huit nationalités sont représentées sur le chantier du tronçon sud confié à entreprise DEME $^{18}$.

Comme au XIX ${ }^{\mathrm{e}}$ siècle, le chantier utilise des engins à la pointe de technologie dont la drague demeure la machine emblématique. Au XIX ${ }^{\mathrm{e}}$ siècle, 78 dragues avaient été construites pour le chantier ; en une dizaine d'années, elles dégagèrent 74 millions de mètres cubes de déblais ${ }^{19}$. En 2014, les nouveaux engins sont capables d'extraire en deux mois la même quantité. Les dragues alors utilisées ont pour ancêtres celles utilisées en 1869 mais avec une capacité d'excavation multipliée par cent. $75 \%$ des dragues existantes à l'échelle mondiale ont été mobilisées pour ce nouveau chantier, soit 45 dragues, travaillant jour et nuit. Les dragues les plus performantes disposent de ponts hydrauliques qui injectent de l'eau pour extraire les sédiments. Parmi elles se distingue la drague à pelle retro-excavatrice, machine très puissante dont trois pieux permettent de stabiliser le ponton sur lequel se dresse une pelleteuse qui creuse le lit du canal et dépose les déblais dans une barge amarrée le long de la drague. Cette drague travaille avec n'importe quel type de sol, ce qui est bien adapté au canal dont les sols sont essentiellement sablonneux et argileux.

Autre drague emblématique, la drague aspiratrice a fait son apparition sur le canal dans années 1930. Depuis, les nouvelles générations représentent des engins de plus de 200 mètres de long qui peuvent aspirer 10000 mètres cubes en une heure, ce qui aurait requis environ 10000 ouvriers au $\mathrm{XIX}^{\mathrm{e}}$ siècle. La drague aspiratrice peut extraire les matériaux grâce à d'énormes tuyaux traînant jusqu'au fond du canal et munis d'une sorte de tête d'aspirateur géante. Sa particularité est d'aspirer tout en avançant, ce qui représente un avantage conséquent dans le tronçon où les travaux ne doivent pas interrompre la navigation.

Pour la partie des bancs rocheux, les ingénieurs ont eu recours aux dragues désagrégatrices, une machine spécialement adaptée à ce type de sol. Au total, 25 dragues désagrégatrices ont été utilisées sur le nouveau chantier. La drague D'Artagnan de l'entreprise DEME a été l'une des premières mobilisée depuis un chantier situé dans la péninsule de Yamal en Russie.

18. Témoignage de Pierre Catteau, responsable de chantier pour l'entreprise de dragage DEME dans le film Le Chantier du canal de Suez, 2016.

19. Le Canal, n 12, juin 1954, p. 2. 
Avec ses 38000 chevaux, elle compte parmi les plus puissantes au monde. La drague se positionne et se fixe au sol grâce à d'énormes pieux et tourne autour d'un axe à l'aide de treuils latéraux reliés à des ancres géantes ; à l'avant du navire, une fraise d'environ trois mètres de diamètre désagrège les roches, sous l'eau comme sur les rives. Les matériaux sont ensuite aspirés par des pompes hydrauliques, puis refoulés par des conduites flottantes. Quand il est possible de déverser les déblais dans des zones immergées, la conduite reliée à la désagrégatrice est arrimée à un radeau équipé d'un système d'épandage continu. L'autre option pour évacuer les déblais consiste à les placer dans des chalands amarrés à la drague où des volets situés sous la coque les déposent selon la technique du « clapage » dans une vaste zone de déstockage à l'ouest du lac Amer. Une dernière option consiste en la technique " arc-en-ciel » : les matériaux dragués sont jetés en l'air afin de recharger les plages.

Le chantier de 2014 en creusant une nouvelle voie, en modifiant les berges et en créant de vastes espaces de sédimentation, a dessiné de nouveaux paysages. Désormais des montagnes de sable se dressent sur les rives du parcours du tronçon doublé. Sur les 72 kilomètres approfondis, la circulation peut se fait à double sens alors que jusque-là, le canal devenu trop étroit, y rendait obligatoire la circulation alternative.

\section{Conclusion}

Le 6 août 2015, lors de l'inauguration des travaux lancés un an plus tôt, l'Égypte renoue avec les accents triomphateurs du XIX siècle. L'événement se veut international avec la présence des grands chefs d'État. Autre clin d'œil à l'histoire, la France est le pays invité d'honneur de la cérémonie et le président Sissi traverse la voie d'eau fièrement installée à la proue du yacht Mahroussa, rebaptisé El-Horriya en 1956, celui-là même qui avait inauguré le canal en 1869 avec à son bord l'impératrice Eugénie, épouse de Napoléon III. Entre-temps, le canal de Suez a considérablement évolué ; sa longueur de 164 kilomètres en 1869 a été portée à 193 kilomètres en 2015; le canal est désormais doublé sur 113 kilomètres. La largeur de la cuvette mesurée à la surface de l'eau est passée selon le tracé de 54 et 100 mètres en 1869, à 300 et 365 mètres aujourd'hui. Quant à la profondeur de l'eau, elle est passée de 8 à 24 mètres, faisant évoluer le tirant d'eau maximum autorisé de 22 à 66 pieds. Le canal, adapté aux navires d'un tonnage de 
5000 tonnes en 1869, peut désormais accueillir les 240000 tonnes en pleine charge.

Les travaux et les études sur les grandes structures s'attachent plus aux phases spectaculaires des chantiers de conception qu'à leur évolution dans le temps. Pourtant, l'enjeu pour les canaux maritimes consiste à s'adapter en permanence à leur environnement technique et économique, lourde tâche, mais prix à payer pour conserver leur rang parmi les grandes routes du commerce maritime.

\section{Bibliographie}

Le Canal de Suez, bulletin décadaire de la compagnie universelle du canal de Suez, 1872-1939.

Le Canal de Suez. Documents statistiques établis par la compagnie du canal de Suez en vue de la XVII session de l'institut international de statistiques, CUCMS, Paris, 1927.

Le Canal de Suez. Documents statistiques, CUCMS, Paris, 1936-1937.

Le Canal de Suez. Notes, tableaux et planches, CUCMS, Paris, 1908, 1937, 1950.

Le Chantier du canal de Suez, chantier de l'extrême, film écrit par Johanna Gauterie et coécrit par Richard Poisson, production Eclectic Press, 2016, diffusé sur RMC Découverte, le 26 janvier 2017.

Loan Agreement, between International Bank for Reconstruction and Development and Suez Canal Authority, 22 décembre 1959.

Loan Agreement between International Bank of Reconstruction and Development and Suez Canal Authority, 20 décembre 1974.

Montel Nathalie, Le Chantier du canal de Suez, 1859-1869. Une histoire des pratiques techniques, Presses de l'École nationale des Ponts et Chaussées, Paris, 1998.

Piquet Caroline, Histoire du canal de Suez, Perrin, Paris, 2009.

Piquet Caroline, Le Canal de Suez, une voie maritime pour l'Égypte et le monde, ErickBonnier, Paris, 2018.

Suez Canal Authority, Suez Canal Yearly Report, Ismaïlia, 1991-2016. 
Le canal de Suez face aux évolutions de la marine marchande

\section{L'autrice}

Agrégée et docteur en histoire, Caroline Piquet est maîtresse de conférences en histoire contemporaine à Sorbonne-Université. Spécialiste de l'histoire économique et sociale de la Méditerranée et du Moyen-Orient, elle a notamment publié Les pays du Golfe, de la perle à l'économie de la connaissance. Les nouvelles terres du libéralisme, Paris, Armand Colin, 2013 et Histoire du canal de Suez, Paris, Perrin, 2009 avec une réédition augmentée : Le canal de Suez, une voie maritime pour l'Égypte et le monde, Paris, Erick Bonnier, 2018. 